\title{
BMJ Open CareTrack Aged: the appropriateness of care delivered to Australians living in residential aged care facilities: a study protocol
}

\author{
Peter D Hibbert, ${ }^{1,2}$ Louise K Wiles, ${ }^{1,2}$ Ian D Cameron, ${ }^{3}$ Alison Kitson, ${ }^{4}$ \\ Richard L Reed, ${ }^{5}$ Andrew Georgiou, ${ }^{1}$ Len Gray, ${ }^{6}$ Johanna Westbrook, ${ }^{1}$ \\ Hanna Augustsson, ${ }^{1}$ Charlotte J Molloy, ${ }^{1,2}$ Gaston Arnolda, ${ }^{1}$ Hsuen P Ting, ${ }^{1}$ \\ Rebecca Mitchell, ${ }^{\oplus 1}$ Frances Rapport, ${ }^{\oplus}$ Susan J Gordon, ${ }^{4}$ William B Runciman, ${ }^{1,2}$ \\ Jeffrey Braithwaite 1
}

To cite: Hibbert PD, Wiles LK, Cameron ID, et al. CareTrack Aged: the appropriateness of care delivered to Australians living in residential aged care facilities: a study protocol. BMJ Open 2019;9:e030988. doi:10.1136/ bmjopen-2019-030988

- Prepublication history and additional material for this paper are available online. To view these files, please visit the journal online (http://dx.doi. org/10.1136/bmjopen-2019030988).

Received 10 April 2019

Revised 27 May 2019

Accepted 28 May 2019
Check for updates

(C) Author(s) (or their employer(s)) 2019. Re-use permitted under CC BY-NC. No commercial re-use. See rights and permissions. Published by BMJ.

For numbered affiliations see end of article.

Correspondence to

Professor Jeffrey Braithwaite; jeffrey.braithwaite@mq.edu.au

\section{ABSTRACT}

Introduction The aged population is increasing rapidly across the world and this is expected to continue. People living in residential aged care facilities (RACFs) represent amongst the sickest and frailest cohort of the aged population, with a high prevalence of chronic conditions and complex comorbidities. Given the vulnerability of RACF residents and the demands on the system, there is a need to determine the extent that care is delivered in line with best practice ('appropriate care') in RACFs. There is also a recognition that systems should provide care that optimises quality of life (QoL), which includes support for physical and psychological well-being, independence, social relationships, personal beliefs and a caring external environment. The aims of CareTrack Aged are to develop sets of indicators for appropriate care and processes of care for commonly managed conditions, and then assess the appropriateness of care delivered and QoL of residents in RACFs in Australia.

Methods and analysis We will extract recommendations from clinical practice guidelines and, using expert review, convert these into sets of indicators for 15 common conditions and processes of care for people living in RACFs. We will recruit RACFs in three Australian states, and residents within these RACFs, using a stratified multistage sampling method. Experienced nurses, trained in the CareTrack Aged methods ('surveyors'), will review care records of recruited residents within a 1-month period in 2019 and 2020, and assess the care documented against the indicators of appropriate care. Surveyors will concurrently assess residents' QoL using validated questionnaires.

Ethics and dissemination The study has been reviewed and approved by the Human Research Ethics Committee of Macquarie University (5201800386). The research findings will be published in international and national journals and disseminated through conferences and presentations to interested stakeholder groups, including consumers, national agencies, healthcare professionals, policymakers and researchers.
Strengths and limitations of this study

- Obtaining national agreement on clinical indicators of appropriate care in residential aged care facilities (RACFs) that may be used for clinical point-of-care decision-making and benchmarking.

- Securing population-level data on the appropriateness of care delivered in RACFs for 15 conditions and residents' quality of life (QoL).

- Providing baseline data for ongoing monitoring of appropriateness of care and residents' QoL in RACFs in Australia.

- Selection bias may be introduced by the refusal of consent for the study from some of the randomly selected RACFs.

\section{INTRODUCTION}

The aged population is increasing rapidly across the world. ${ }^{1-4}$ In 2017, $15 \%$ of the Australian population were aged 65 years and older and 2\% were aged 85 years and older. This is projected to increase to $22 \%$ and $4 \%$, respectively, by $2066 .{ }^{5}$ People living in residential aged care facilities (RACFs) represent amongst the sickest and frailest cohort of the ageing population; this manifests with this group having the highest rate of disability in the Australian population. ${ }^{6}$ The prevalence of chronic conditions among RACF residents is estimated to be $80 \%$ for sensory loss, $60 \%$ for dementia, $40 \%-80 \%$ for chronic pain, $50 \%$ for urinary incontinence, $45 \%$ for sleep disorder and 30\%-40\% for depression. ${ }^{6}$ In the 2017-2018 financial year, over 215000 people entered RACFs, representing an increase of $31 \%$ in admissions over the last decade. ${ }^{7}$ People in RACFs have increasingly high levels of dependency and more complex 
medical needs than other similar cohorts in relation to chronic illness, physical disability and dementia. ${ }^{6}$

The current aged care system needs to be ready for these future demands while providing high-quality care. Consequently, there is a need to understand if appropriate care (defined as care in line with evidence-based or consensus-based guidelines) in RACFs can be delivered reliably. ${ }^{9}$ Two recent large-scale studies on the quality of care showed that, for adults living in Australia, appropriate care was offered, on average, $57 \%$ of the time $^{10}$ and for children, $60 \%$ of the time. ${ }^{11}$ However, the level of appropriate care provided to people living in Australian RACFs and their corresponding cohorts in other countries is largely unknown. Some studies on single conditions undertaken in a limited number of RACFs have shown that only $13 \%-41 \%$ of residents at risk of falls receive vitamin D supplementation ${ }^{12}{ }^{13} ; 34 \%$ of residents received appropriate non-pharmacological pain management ${ }^{14} ; 57 \%$ of diabetic residents who were actively managed (ie, with insulin or oral medication) and had a haemoglobin A1c test performed every 6 months ${ }^{15}$ and $50 \%$ were malnourished with $20 \%$ of these severe. ${ }^{16}$ However, a study on the appropriateness of care for multiple conditions in multiple RACFs across more than one state, using a standardised methodology, has not been undertaken.

There is a growing consensus that healthy ageing is more than just the absence of disease or infirmity but is a state of physical, mental and social well-being, with a focus on optimising opportunities for health, safety, social participation and security. ${ }^{4}$ Measuring the impact of care provided in RACFs, therefore, needs to begin with the recognition that quality of life (QoL) is a multidimensional concept, which views a person's health status in the context of support for physical and psychological well-being, independence, social relationships, positive personal beliefs and a caring environment. ${ }^{16}$ The Organisation for Economic Co-operation and Development, reporting on improving the quality of aged care services, has focused attention on deficiencies in the aged care sector common to many countries. This includes overemphasis on measuring structural inputs (eg, resource utilisation) to the disadvantage of measures of client outcomes and satisfaction and QoL. ${ }^{17}{ }^{18}$ While there is some evidence to suggest a link between quality of care and QoL, with factors, such as depression, ${ }^{19} 20$ hydration and falls, ${ }^{20}$ being associated with poorer resident QoL, study findings are limited by the use of small, non-random samples, and a lack of process measures for quality of care.

Given the pressures on the system and the need for a sustainable and high-quality residential aged care model, it is vital and urgent to assess to what extent care delivered to Australians in RACFs is in line with the evidence (appropriate care) and to assess QoL. The CareTrack Aged study will address this with three aims:

1. To obtain national agreement on sets of indicators for appropriate care of commonly managed conditions and processes of care in RACFs in Australia.

\section{Box 1 CareTrack Aged study definitions}

- A resident is a person aged 65 years and older living in a residential aged care facility (RACF).

- Condition refers to acute (eg, pressure injuries and hip fractures) and chronic conditions (eg, dementia and incontinence) or care processes (eg, medication management and oral and dental care).

- Healthcare provider includes any healthcare professional offering services to residents within an RACF, and whose scope of practice is covered by identified clinical practice guideline (CPG) recommendations.

- Appropriate care is care considered to be evidence-based or consensus-based by a panel of experts in Australia in the context in which it was delivered in the years 2019 and 2020.

- A clinical indicator is a measurable component of a standard or guideline, with explicit criteria for inclusion, exclusion, time frame and setting. In the context of this study, an indicator is relevant for Australian practice during 2019 and 2020. Each indicator is scored as to whether eligible processes for prevention, monitoring or treatment of a condition have been carried out by answering 'yes' or 'no'. Phases of care include admission, screening, diagnosis, assessment, treatment, ongoing management and prevention.

- An encounter is an interaction between a resident and a healthcare provider defined by the inclusion criteria of the clinical indicators.

- Adherence with indicators is expressed as the percentage of eligible encounters at which appropriate care was received.

- A surveyor is a person with appropriate clinical and audit experience who has been trained and accredited for this study to review care records in relation to the care indicators.

2. To measure the appropriateness of care delivered in RACFs in Australia.

3. To assess the QoL of residents in RACFs in Australia.

\section{METHODS AND ANALYSIS}

The CareTrack Aged protocol is based on the previously validated methodology used in the American ${ }^{21}$ and our Australian studies of the quality of healthcare, CareTrack Australia $^{910}$ and CareTrack Kids. ${ }^{1122}{ }^{23}$ We will develop a set of indicators relevant to common conditions and processes of care for people living in RACFs, recruit RACFs and residents within RACFs, review care records for a 1-month period in 2019 and 2020, and assess these against indicators of appropriate care, while concurrently assessing residents' QoL (box 1). The study will be conducted in seven stages (figure 1).

\section{Stage 1. Selecting conditions and developing indicators Selecting conditions}

Fifteen conditions relevant to estimating appropriateness of care at the population level (table 1 ) were identified from published research, ${ }^{19} 2024$ prevalence and burden of disease data, ${ }^{16}{ }^{25}$ clinical practice guidelines (CPGs) and indicator sets relevant to RACF settings. The importance of assessing the appropriateness of care provision unrelated to specific medical conditions, such as routine care processes (eg, oral healthcare), was recognised by the research team and these were incorporated into the list of conditions and care processes. The 


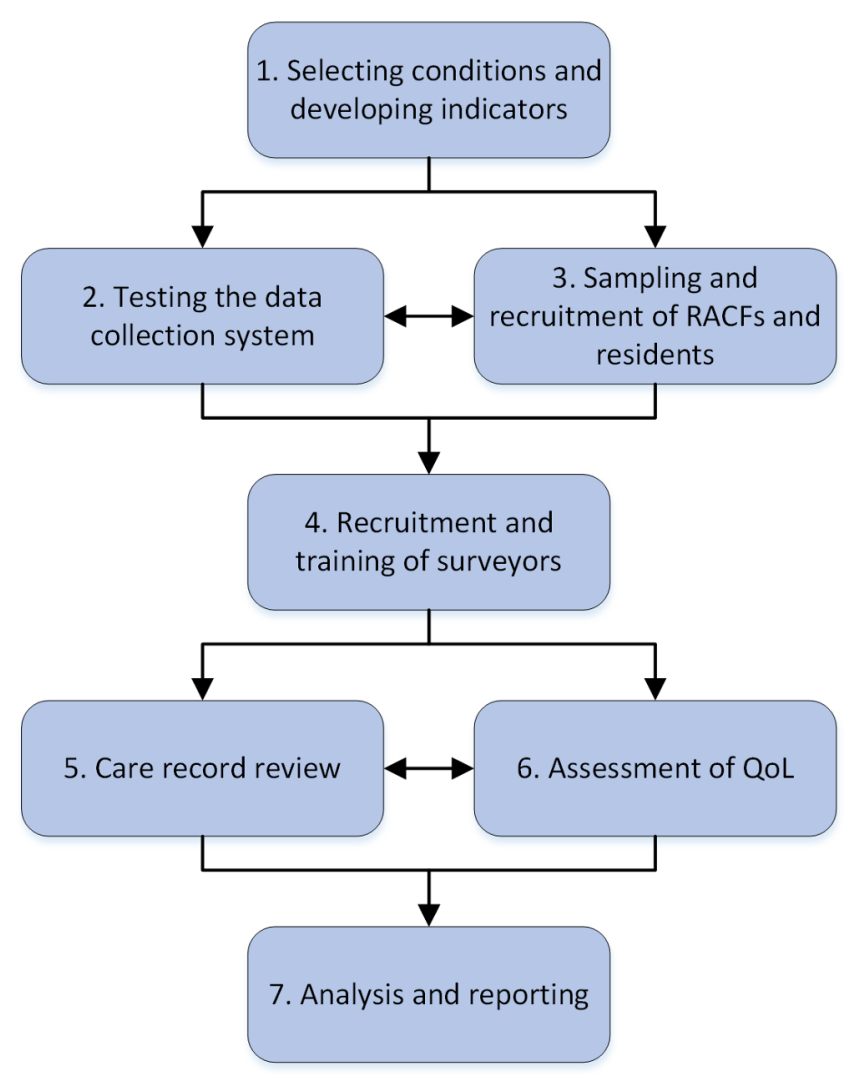

Figure 1 Schematic diagram of stages in the CareTrack Aged study. RACFs, residential aged care facilities; QoL, quality of life.

defined scope for each condition and care process was informed and further refined using prevalence data, and the approaches of related CPGs, ${ }^{26-30}$ and indicator sets, such as the interRAI ${ }^{31}$ and the Assessing Care of Vulnerable Elders-3 Quality Indicators. ${ }^{32-34}$ Condition and care process labels were assigned in accordance with contemporary terminology in the field and the expected likelihood and level of documentation of conditions or care processes in resident care records, such as dementia and delirium being grouped under the more commonly used term 'cognitive impairment' (table 1). Online supplementary appendix 1 shows the estimated prevalence of conditions or care processes in RACF.

\section{Developing indicators}

The definition of a clinical indicator is presented in box $1 .^{1022}$ The source of indicators for selected conditions will be recommendations in CPGs. The CPGs will be identified by a systematic search, developed in conjunction with an academic librarian at the University of South Australia, using medical subject heading $(\mathrm{MeSH})$ and keyword terms in the peer-reviewed literature $^{35}$ and targeted searches of national and international sites, for example, the Royal Australian College of General Practitioners, the National Health and Medical Research Council (NHMRC) and the UK's National Institute for Health and Care Excellence. We will also draw on the indicators developed by the interRAI collaborative, which have been found to have robust psychometric properties. $^{36-38}$

Candidate recommendations will be extracted from the CPGs and recorded in a spreadsheet, including level or grade of evidence. Duplicate recommendations will be merged. Recommendations will be excluded based on the strength or certainty of their wording, such as citing weak evidence for the recommendation or the use of words, such as 'may' or 'could'; low likelihood of the information being documented in residents' records and guiding statements without recommended actions. The extracted recommendations will be collated and used to inform the content and format of proposed clinical indicators. All indicators will be written using a structured and standardised format starting with the inclusion criteria followed by the adherence action. Indicators will be arranged according to phases of care (ie, admission, screening, diagnosis, assessment, treatment, ongoing management and prevention) and will address both underuse and overuse of care provision.

\section{Review of the indicators}

Indicators for each condition will be embedded within a secure online survey for open, transparent and formal review by national experts (such as clinicians and researchers with appropriate experience) and consumer representatives and groups. The aim is to get a minimum of five independent expert reviews for each condition. Experts will rate the indicators on a nine-point Likert scale for their representativeness for appropriate aged care delivered in RACFs during 2019 and 2020, and rank them according to their acceptability, feasibility and clinical impact. ${ }^{22}$ A clinical champion for each condition, appointed from within the expert group or by drawing on their professional networks, will follow-up and manage external reviewers' ratings and make final recommendations regarding the inclusion, content, structure and format of indicators. A final list of indicators will be collated to represent appropriate care for those living in RACFs for selected conditions during 2019 and 2020.

\section{Stage 2. Testing the data collection system}

A web-based tool for data collection has been modified from that used in the CareTrack Australia ${ }^{10}$ and CareTrack $K_{i d s^{11}}$ studies. The tool supports secure data access, encryption, off-line data collection and database synchronisation to mitigate against potential problems with firewalls and poor internet connectivity. In this stage, the data collection system and processes will be piloted by a trained surveyor in five RACFs with the support of the research team. The testing will include accessibility of residents' records; the structure and depth of information recorded; time taken to access and review records; data collection workflow; usability of the records to score indicators; processes for inter-rater reliability (IRR) testing; frequency of the conditions treated and care processes documented. Findings from the pilot study will be used to help to determine the types of problems 
Table 1 Candidate conditions and processes of care, and relevant CPGs supporting their inclusion

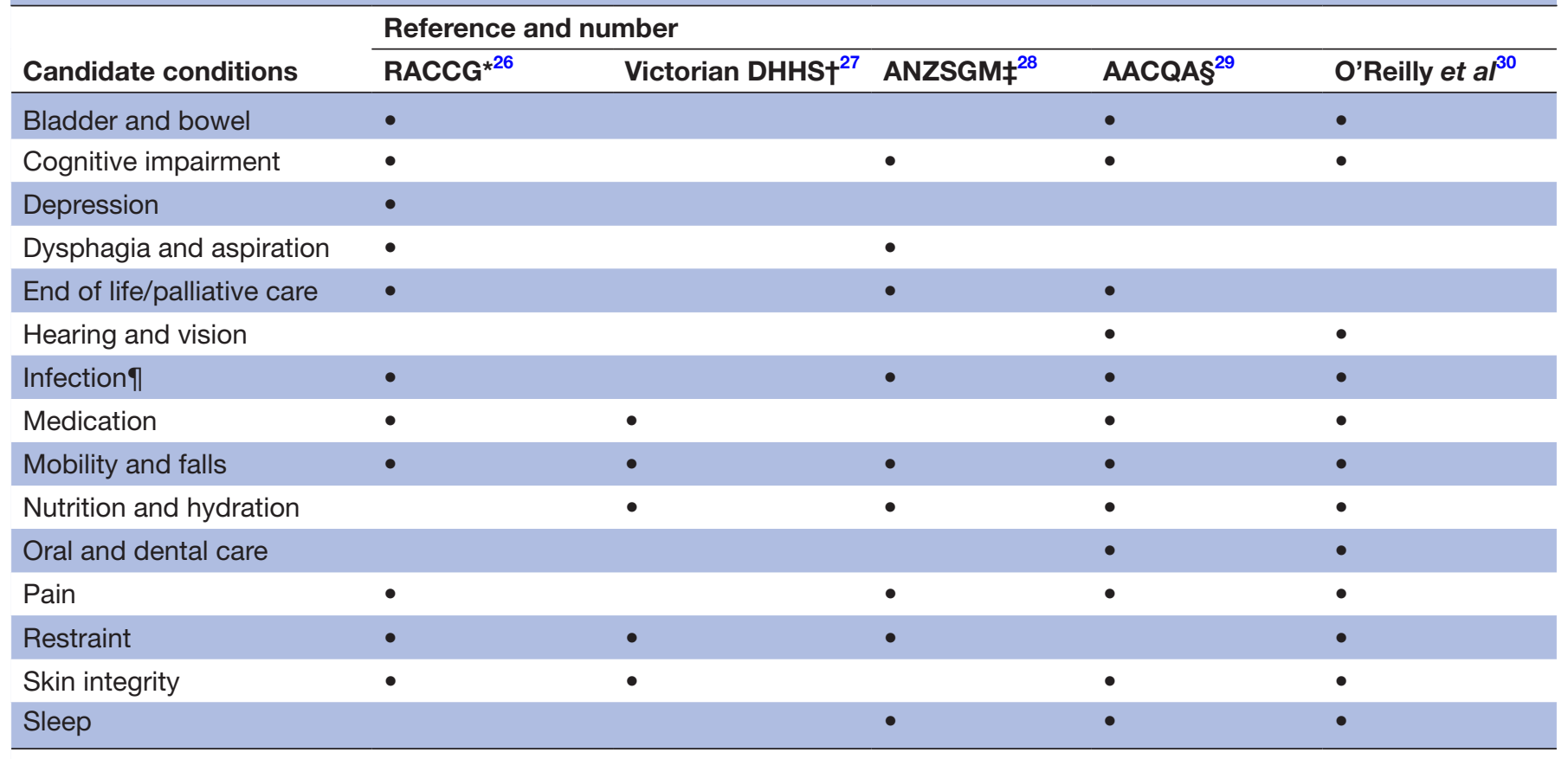

*Royal Australian College of General Practitioners.

†Department of Health and Human Services.

$\ddagger$ Australian and New Zealand Society of Geriatric Medicine.

$\S$ Australian Aged Care Quality Agency.

ๆlncludes respiratory infections (influenza and pneumonia), vaccination and urinary tract infections.

CPGs, clinical practice guidelines.

that may be encountered and will inform the final selection of conditions, their indicators and the logistical and practical aspects of recruiting RACFs and residents, of accessing records, and of extracting, recording, storing and analysing the data. The data obtained from the pilot will not be included in the main results.

\section{Stage 3. Sampling and recruitment}

Data on the appropriateness of care will be collected from three states in Australia-New South Wales (NSW), Queensland (Qld) and South Australia (SA). The sampling frame for RACFs will be the list maintained by the Australian Aged Care Quality Agency (AACQA, now the Aged Care Quality and Safety Commission), ${ }^{39}$ which groups RACFs into Aged Care Planning Regions (ACPR). ${ }^{40}$ The list includes the number of licensed beds at each facility, the five-category Australian Standard Geographical Classification of Remoteness Areas (ie, major cities, inner regional, outer regional, remote and very remote) and ownership type (eg, not for profit, private and government). The three selected states have $59 \%$ of RACFs and $62 \%$ of residential beds in Australia. For logistical practicality, RACFs in the following categories will be removed prior to sampling:

1. RACFs with $<20$ approved beds.

2. RACFs located in remoteness areas classified as remote or very remote.

3. Major city RACFs in ACPRs with $<500$ major city beds.
4. Inner regional RACFs in ACPRs with <200 inner regional beds.

5. Outer regional RACFs in ACPRs with $<100$ outer regional beds.

Using 2017 AACQA service list data, there are 1571 RACFs in the three selected states with 123520 approved beds. Removing the ineligible RACFs removes 80 RACFs $(5.1 \%)$ and 2461 approved beds (2.0\%). After these exclusions, the sampling frame will contain 1491 RACFs containing 121059 approved beds.

A stratified multistage sampling design with five stages will be used, defined as follows:

1. States will be treated as strata and allocated sampling units proportional to the number of approved beds they contain (NSW 56.2\%, Qld 29.7\% and SA $14.1 \%$ ).

2. Within each state, ACPRs will be organised into five substrata: (1) Major city RACFs; (2) Major city and inner regional RACFs; (3) Inner regional RACFs; (4) Inner and outer regional RACFs and (5) Outer regional RACFs. Each substratum will be allocated a quota of planning regions and facilities to be sampled, the latter being allocated approximately proportional to the number of approved beds in the substratum.

3. Within each substratum, the allocated number of ACPRs will be selected randomly, with the probability of selection being proportional to the number of approved beds in the ACPR. 
4. Within each selected ACPR, facilities will be randomly selected, with the probability of selection proportional to the number of approved beds in the facility, sequentially ordered from first to last and approached for approval starting at the top-if consent is denied, the next facility will be approached until an allocated quota is reached.

5. Within each consented facility, the eligible residents will be listed in a random order and approached sequentially until a quota of 10 consented individuals is reached. The surveyor will obtain written consent from the resident. A threshold resident Psychogeriatric Assessment Scale-Cognitive Impairment (PAS-Cog) score of 12 will be used to determine cognitive impairment. ${ }^{41}$ Residents who are cognitively impaired will be consented to participate in CareTrack Aged through a nominated proxy (ie, carer and family member). Eligibility criteria for residents are aged 65 years and older; resident in the RACF at least 30 days and English speaking.

To achieve a precision of $\pm 5 \%$ around the estimate of adherence for each condition or care process under examination with $95 \%$ confidence, 384 eligible indicator encounters will need to be assessed. Conservatively, assuming a point estimate of adherence of $50 \%$, an intracluster correlation coefficient of 0.3 for RACFs and only one eligible indicator per sampled resident, 143 RACFs would need to be sampled, with 10 residents sampled per RACF, to ensure broad cover. To improve the representativeness of the sample, we intend sampling around 150 RACFs, which will provide a total of 1500 individuals and their care records for review.

\section{Stage 4. Recruitment and training of surveyors}

Experienced registered healthcare professionals will be recruited as surveyors to review care records. As part of the recruitment process, prospective surveyors will perform a test that involves assessment of artificially constructed care records, which will be designed by the research team. After being recruited, surveyors will receive training, including further assessment of artificially constructed care records; education about included conditions, such as the evidence in the literature and in CPGs; indicator inclusion and exclusion criteria; assessment and management procedures; IRR testing; and database orientation and training. Each surveyor will be provided with a detailed manual that outlines the conditions, indicators, definitions, abbreviations, criteria and processes for arranging and conducting record reviews.

IRR testing will be conducted prior to data collection for all the surveyors against one of the experienced CareTrack Aged researchers. Artificial records will be used for coding and IRR testing. Before being approved to collect data, each surveyor must achieve a minimum Kappa score of 0.7 against the gold standard for (i) correctly identifying the eligibility of an indicator ('Yes' vs 'No') and (ii) correctly scoring the indicator ('Yes' vs 'No'). After, all surveyors have met the minimum threshold, a series of complete real records (ie, in excess of 500 indicators) will be assessed by all qualified surveyors to estimate final Kappa scores.

\section{Stage 5. Care records review}

During the data collection, surveyors will review the previous 1 month of care records for 10 residents per participating RACF. The surveyors will compare the content in the care records against the indicators in order to assess whether the adherence actions have been met. Data will be extracted by surveyors in an explicit criterion-based record review and recorded with the data collection tool that was developed for the CareTrack Australia ${ }^{10}$ and CareTrack Kids ${ }^{11}$ studies.

\section{Stage 6. Assessment of QoL}

Residents who consent to the care record review will also be asked to participate in a face-to-face interview to assess their QoL. Surveyors will recruit the residents and undertake the interviews. For cognitively impaired residents, a nominated family member or carer will act as a proxy for the assessment. The instruments that will be used for assessing QoL are both the interRAI subjective quality of life (SQOL) ${ }^{42}$ and a standardised instrument for measuring health outcomes $(\mathrm{Q}-5 \mathrm{D}-5 \mathrm{~L}$, the 5-level version of the EQ-5D) ${ }^{43}$ for RACF residents without cognitive impairment (ie, PAS-Cog<12) or the Quality of Life in Alzheimer's Disease (QoL-AD) ${ }^{44}$ for residents deemed to have cognitive impairment (ie, PAS-Cog score of 12 or more).

\section{Stage 7. Analysis}

In addition to producing a set of indicators that represent appropriate care in RACFs, the study will produce three outputs: (i) estimates of the percentage of appropriate care in RACFs; (ii) estimates of QoL scores of residents living in RACFs and (iii) exploratory analysis of the association between appropriateness of care and QoL scores. Analysis will be performed in SAS/STAT software V.9.4.

\section{Estimates of percentage of appropriate care}

Indicators will be aggregated to estimate the percentage of appropriate care for each condition or care process, and to estimate the percentage of appropriate care overall. The percentage of appropriate care is the total number of 'yes' responses divided by the total number of eligible indicators, adjusted with sampling weights. Exact $95 \%$ CIs will be generated by the modified Clopper-Pearson method and variance by Taylor series linearisation.

\section{Estimates of QoL scores}

Individual QoL scores will be calculated for each instrument (interRAI SQoL and EQ-5D-5L for residents without cognitive impairment and the QoL-AD for residents with cognitive impairment). ${ }^{42-44}$ Means and $95 \%$ CIs will be calculated by level for each factor (eg, individual characteristics, facility characteristics, state and geographical location), adjusted by sampling weights. 
Exploratory analysis of the association between appropriateness of care and QoL scores

The relationship between the appropriateness of care and the QoL scores will be explored, adjusted with multiple factors. Mixed effects models will be used to take account of repeated measures within clusters. ${ }^{19}$

\section{PATIENT AND PUBLIC INVOLVEMENT}

This research study has a policy and advisory group that includes consumer representatives who were involved in its design and ongoing conduct.

\section{ETHICS AND DISSEMINATION Ethics}

Site-specific approvals will be sought prior to the recruitment of participants and before undertaking the care record reviews and QoL assessments.

\section{Dissemination}

The research findings will be published in international and national journals and disseminated through conferences and presentations to the various stakeholder groups, including consumers, healthcare professionals, policymakers, RACF organisations and facilities, and researchers.

\section{DISCUSSION}

Population-level information regarding the appropriateness of care delivery for RACF residents is not available in Australia nor in other countries. This is clearly a gap in infrastructure and knowledge about the performance of care delivered by the system. On the basis of the results of CareTrack Aged, empirically driven priorities for improvement at a systems level can be set by the federal and state governments, and peak bodies and colleges to address improving the level of appropriate care for commonly occurring clinical processes and conditions. Care systems will be unaffordable unless funds are diverted to more appropriate care with reduced adverse events, less waste, more efficient use of limited resources and towards care, which optimises opportunities for health, safety, social participation and security.

Healthy ageing is indeed more than just the absence of disease or infirmity and more a complex mix of physical, mental and social well-being factors, optimising opportunities for health, participation and security. Our intention to measure the impact of care provided in aged care settings, therefore, needs to begin with the recognition that QoL is as important as the delivery of evidence-based or consensus-based care and while they are two sides of the same coin, the two concepts may be relatively independent of each other.

\section{Author affiliations}

${ }^{1}$ Australian Institute of Health Innovation, Macquarie University, Sydney, New South Wales, Australia
${ }^{2}$ Australian Centre for Precision Health, Cancer Research Institute, School of Health Sciences, University of South Australia, Adelaide, South Australia, Australia ${ }^{3}$ John Walsh Centre for Rehabilitation Research, Kolling Institute, Faculty of Medicine and Health, University of Sydney, St Leonards, New South Wales, Australia ${ }^{4}$ College of Nursing and Health Sciences, Flinders University, Bedford Park, South Australia, Australia

${ }^{5}$ Flinders University General Practice and Primary Health Care, College of Medicine and Public Health, Flinders University, Bedford Park, South Australia, Australia

${ }^{6}$ Centre for Health Services Research, Faculty of Medicine, University of Queensland, Herston, Queensland, Australia

Contributors JB and PDH initiated the project and led the NHMRC grant proposal. $\mathrm{JB}, \mathrm{PDH}, \mathrm{IC}, \mathrm{AK}, \mathrm{RR}, \mathrm{AG}$ and $\mathrm{LG}$ co-led all design aspects and shared in the development of the protocol and the initial drafting of the grant application and protocol. Drawing on the JB, PDH grant proposal, HA, LKW, GA and HPT did the first drafting of the protocol manuscript. JW, CJM, RM, FR, SJG and WBR helped to write the grant proposal, protocol and manuscript, and all the authors reviewed all revisions.

Funding This work was supported by a National Health and Medical Research Council Project (Grant No.: 1143223).

Competing interests None declared.

Patient consent for publication Not required.

Ethics approval Human Research Ethics Committee (HREC) approval has been granted from Macquarie University (5201800386).

Provenance and peer review Not commissioned; peer reviewed for ethical and funding approval prior to submission.

Open access This is an open access article distributed in accordance with the Creative Commons Attribution Non Commercial (CC BY-NC 4.0) license, which permits others to distribute, remix, adapt, build upon this work non-commercially, and license their derivative works on different terms, provided the original work is properly cited, appropriate credit is given, any changes made indicated, and the use is non-commercial. See: http://creativecommons.org/licenses/by-nc/4.0/.

\section{REFERENCES}

1. Guerin B, Hoorens S, Khodyakov D, et al. A growing and ageing population: Global societal trends to 2030: Thematic report 1. Santa Monica: RAND Corporation 2015.

2. Braithwaite J, Vincent C, Nicklin W, et al. Coping with more people with more illness. Part 2: new generation of standards for enabling healthcare system transformation and sustainability. Int J Qual Health Care 2019;31:159-63.

3. Amalberti R, Vincent C, Nicklin W, et al. Coping with more people with more illness. Part 1: the nature of the challenge and the implications for safety and quality. Int J Qual Health Care 2019;31:154-8.

4. World Health Organization. World Report on Ageing and Health. Geneva, Switzerland: World Health Organization, 2015.

5. Australian Bureau of Statistics. PopulationProjections, Australia, 2017 (base) - 2066. 2018 https://www.abs.gov.au/ausstats/abs@.nsf/ 0/5A9C0859C5F50C30CA25718C0015182F?Opendocument.

6. Flicker L. Clinical issues in aged care: managing the interface between acute, subacute, community and residential care. Aust Health Rev 2002;25:136-9.

7. Australian Insitute of Health and Welfare. Admissions into aged care. 2017 http://www.gen-agedcaredata.gov.au/Topics/Admissions-intoaged-care (accessed 22 Nov 2018).

8. Dougherty D, Conway PH. The "3T's" road map to transform US health care: the "how" of high-quality care. JAMA 2008;299:2319-21.

9. Hunt TD, Ramanathan SA, Hannaford NA, et al. CareTrack Australia: assessing the appropriateness of adult healthcare: protocol for a retrospective medical record review. BMJ Open 2012;2:e000665.

10. Runciman WB, Hunt TD, Hannaford NA, et al. CareTrack: assessing the appropriateness of health care delivery in Australia. Med J Aust 2012;197:100-5.

11. Braithwaite J, Hibbert PD, Jaffe A, et al. Quality of health care for children in Australia, 2012-2013. JAMA 2018;319:1113-24.

12. Francis-Coad J, Etherton-Beer C, Bulsara C, et al. Using a community of practice to evaluate falls prevention activity in a residential aged care organisation: a clinical audit. Aust Health Rev 2017;41:13-18. 
13. Ward JA, Harden M, Gibson RE, et al. A cluster randomised controlled trial to prevent injury due to falls in a residential aged care population. Med J Aust 2010;192:319-22.

14. Savvas SM, Toye CM, Beattie ER, et al. An evidence-based program to improve analgesic practice and pain outcomes in residential aged care facilities. J Am Geriatr Soc 2014;62:1583-9.

15. Haines HM, Bannon-Murphy $\mathrm{H}$, Amos $\mathrm{T}$, et al. Prevalence and management of diabetes in residential aged care facilities in northeast Victoria, Australia. Aust Fam Physician 2016;45:908-11.

16. Banks M, Ash S, Bauer J, et al. Prevalence of malnutrition in adults in Queensland public hospitals and residential aged care facilities. Nutrition \& Dietetics 2007;64:172-8.

17. Organisation for Economic Co-operation and Development (OECD). Long-Term Care for Older People: OECD study (2001-2004). Paris, France: OECD 2005

18. Organisation for Economic Co-operation and Development (OECD). A good life in old age? Monitoring and improving quality in long-term care. Paris, France: OECD, 2013.

19. Beerens HC, Sutcliffe C, Renom-Guiteras A, et al. Quality of life and quality of care for people with dementia receiving long term institutional care or professional home care: the European RightTimePlaceCare study. J Am Med Dir Assoc 2014;15:54-61.

20. Courtney M, O'Reilly M, Edwards $\mathrm{H}$, et al. The relationship between clinical outcomes and quality of life for residents of aged care facilities. Aust J Adv Nurs 2009;26:49.

21. McGlynn EA, Asch SM, Adams J, et al. The quality of health care delivered to adults in the United States. N Engl J Med 2003;348:2635-45

22. Wiles LK, Hooper TD, Hibbert PD, et al. CareTrack Kids-part 1. Assessing the appropriateness of healthcare delivered to Australian children: study protocol for clinical indicator development. BMJ Open 2015;5:e007748.

23. Hooper TD, Hibbert PD, Mealing N, et al. CareTrack Kids-part 2. Assessing the appropriateness of the healthcare delivered to Australian children: study protocol for a retrospective medical record review. BMJ Open 2015;5:e007749.

24. Stafford AC, Alswayan MS, Tenni PC. Inappropriate prescribing in older residents of Australian care homes. J Clin Pharm Ther 2011;36:33-44.

25. Flicker L, Mead K, Maclnnis RJ, et al. Serum vitamin $D$ and falls in older women in residential care in Australia. J Am Geriatr Soc 2003:51:1533-8.

26. Royal Australian College of General Practitioners. Medical care of older persons in residential aged care facilities. 4th ed. South Melbourne, Australia: RACGP, 2006.

27. Victorian Department of Health and Human Services. Quality indicators in public sector residential aged care services. Resource materials. Melbourne, Australia 2015.
28. Australian and New Zealand Society of Geriatric Medicine. Position Statement 12 - Dysphagia and Aspiration for Geriatric Medicine 2015 http://www.anzsgm.org/posstate.asp (Accessed Feb 2019).

29. Australian Aged Care Quality Agency. About accreditation. Canberra, Australia: Australian Government. http://www.aacqa.gov.au/for-thepublic/for-providers. (Accessed Jan 2019).

30. O'Reilly M, Courtney M, Edwards $\mathrm{H}$, et al. Clinical outcomes in residential care: setting benchmarks for quality. Australas $J$ Ageing 2011;30:63-9.

31. Bernabei R, Landi F, Onder G, et al. Second and third generation assessment instruments: the birth of standardization in geriatric care. J Gerontol A Biol Sci Med Sci 2008:63:308-13.

32. RAND Corporation. Quality Indicators - ACOVE 3. http://www.rand. org/health/projects/acove/acove3.html (Accessed Nov 22 Nov 2018)

33. Amin A, Besdine RK, Blazer DG, et al. Assessing care of vulnerable elders-3 quality indicators. J Am Geriatr Soc 2007;55 Suppl 2(S2):S464-S87.

34. Wenger NS, Roth CP, Shekelle P. ACOVE Investigators. Introduction to the assessing care of vulnerable elders-3 quality indicator measurement set. J Am Geriatr Soc 2007;55 Suppl 2:S247-S252.

35. McGowan J, Sampson M, Salzwedel DM, et al. Press peer review of electronic search strategies: 2015 guideline statement. J Clin Epidemiol 2016;75:40-6.

36. Hirdes JP, Ljunggren G, Morris JN, et al. Reliability of the interRA suite of assessment instruments: a 12-country study of an integrated health information system. BMC Health Serv Res 2008;8:277.

37. Kim H, Jung YI, Sung M, et al. Reliability of the interRAI Long Term Care Facilities (LTCF) and interRAI Home Care (HC). Geriatr Geronto Int 2015;15:220-8.

38. Onder G, Carpenter I, Finne-Soveri $\mathrm{H}$, et al. Assessment of nursing home residents in Europe: the Services and Health for Elderly in Long TERm care (SHELTER) study. BMC Health Serv Res 2012;12:5.

39. Australian Aged Care Quality Agency. http://www.aacqa.gov.au/ (Accessed 25 Nov 2018).

40. Australian Government Department of Health. Aged care planning region maps. $2018 \mathrm{http} / / /$ agedcare.health.gov.au/publications-andarticles/research-and-statistics/aged-care-planning-region-maps (Accessed 25 Nov 2018).

41. Dyer SM, Liu E, Gnanamanickam ES, et al. Clustered domestic residential aged care in Australia: fewer hospitalisations and better quality of life. Med J Aust 2018;208:433-8.

42. Morris JN, Declercq A, Hirdes JP, et al. Hearing the voice of the resident in long-term care facilities-an internationally based approach to assessing quality of life. J Am Med Dir Assoc 2018;19:207-15.

43. Herdman M, Gudex C, Lloyd A, et al. Development and preliminary testing of the new five-level version of EQ-5D (EQ-5D-5L). Qual Life Res 2011;20:1727-36

44. Thorgrimsen L, Selwood A, Spector A, et al. Whose quality of life is it anyway? The validity and reliability of the Quality of Life-Alzheimer's Disease (QoL-AD) scale. Alzheimer Dis Assoc Disord 2003;17:201-8. 